\title{
Orthogonal decomposition of the sum-symmetry model for square contingency tables with ordinal categories: Use of the exponential sum-symmetry model
}

\author{
Shuji Ando \\ Department of Information and Computer Technology, Faculty of Engineering, \\ Tokyo University of Science, Katsushika-ku, Tokyo, 125-8585, Japan, \\ e-mail: shuji.ando@rs.tus.ac.jp
}

\section{SUMMARY}

In the existing decomposition theorem, the sum-symmetry model holds if and only if both the exponential sum-symmetry and global symmetry models hold. However, this decomposition theorem does not satisfy the asymptotic equivalence for the test statistic. To address the aforementioned gap, this study establishes a decomposition theorem in which the sum-symmetry model holds if and only if both the exponential sum-symmetry and weighted global-sum-symmetry models hold. The proposed decomposition theorem satisfies the asymptotic equivalence for the test statistic. We demonstrate the advantages of the proposed decomposition theorem by applying it to datasets comprising real data and artificial data.

Key words: matched-pairs data, orthogonality, sum-symmetry model, test statistic

\section{Introduction}

Statisticians usually cross-classify matched pairs of ordinal categorical variables to obtain square contingency tables with the same row and column ordinal classifications. Table 1 illustrates one of the most popular datasets of such categorical data. The row and column variables represent the grades of the right and left eyes, with the categories ordered from the lowest (1) to the highest grade (4). This dataset has been analyzed by several statisticians, such as Stuart (1955), Caussinus (1965), McCullagh (1978), Read (1977), Goodman (1979), Bhapkar (1966, 1979), Agresti (1983a,b), and Bishop et al. (2007). They performed analysis using statistical models with a symmetric or asymmetric structure (i) between the probability that an observation 
will fall in the $(i, j)$ th cell of the $C \times C$ table and the probability that an observation will fall in the $(j, i)$ th, for $i<j$, and (ii) between the row and column marginal distributions.

Yamamoto et al. (2013) stated that it is natural to evaluate an individual's vision level as the sum of the right and left eye grades for data such as those in Table 1.

Table 1. Dataset for unaided vision of 7,477 women in Britain; source Stuart (1953).

\begin{tabular}{rrrrrr}
\hline & \multicolumn{5}{c}{ Left eye grades } \\
\cline { 2 - 5 } Right eye grades & $(1)$ & $(2)$ & $(3)$ & $(4)$ & Total \\
\hline$(1)$ & 492 & 179 & 82 & 36 & 789 \\
$(2)$ & 205 & 1772 & 362 & 117 & 2456 \\
$(3)$ & 78 & 432 & 1512 & 234 & 2256 \\
$(4)$ & 66 & 124 & 266 & 1520 & 1976 \\
\hline Total & 841 & 2507 & 2222 & 1907 & 7477 \\
\hline
\end{tabular}

Additionally, Yamamoto et al. (2013) introduced the sum-symmetry (SS) and conditional sum-symmetry (CSS) models, wherein the ratio of the probability with which the sum of the row and column levels is $t$ $(t=3,4, \ldots, 2 C-1)$ when the row level is less than the column level to the probability with which the sum of those levels is $t$ when the row level is greater than the column level is always one in the first case, and is constant in the second case. Yamamoto et al. (2016) introduced the exponential sum-symmetry (ESS) model, wherein the ratio of the probability with which the sum of the row and column levels is $t(t=3,4, \ldots, 2 C-1)$ when the row level is less than the column level to the probability with which the sum of those levels is $t$ when the row level is greater than the column level changes exponentially depending on the sum of the row and column levels. Concerning the applicability of these models, the SS, CSS, and ESS models are useful for evaluating an individual's comprehensive strength, for example through vision, hearing, and grip strength data. Concerning the decomposition of these statistical models, Caussinus (1965) originated the decomposition theorem - the symmetry (S) model holds if and only if both the marginal homogeneity $(\mathrm{MH})$ and quasi-symmetry (QS) models hold - for square contingency tables. Tomizawa and Tahata (2007) showed that the value of the likelihood ratio chi-squared statistic of the $\mathrm{S}$ model is asymptotically equivalent to the sum of those of the $\mathrm{MH}$ and QS models. 
Yamamoto et al. (2013) presented a decomposition theorem in which the SS model holds if and only if both the CSS and global symmetry (GS) models hold, and showed that the value of the likelihood ratio chi-squared statistic of the SS model is equal to the sum of those of the CSS and GS models.

In this context, it must be noted that Yamamoto et al. (2016) presented a decomposition theorem wherein the SS model holds if and only if both the ESS and GS models hold. However, this decomposition theorem does not satisfy the asymptotic equivalence for the test statistic. This is because the ESS model is different from the CSS model. To address the aforementioned gap, this study provides a new decomposition theorem that satisfies the asymptotic equivalence for the test statistic. In other words, we aim to identify a model that must satisfy the asymptotic equivalence for the test statistic.

The rest of this paper is organized as follows. Section 2 provides a new decomposition theorem for the SS model using the ESS model and shows the orthogonality of the test statistics for the proposed decomposition theorem. Section 3 demonstrates the advantages of the proposed decomposition theorem by applying it to datasets consisting of the real data in Table 1 and of artificial data. We close with concluding remarks in section 4 .

\section{Orthogonal decomposition of the sum-symmetry model}

Let $X$ and $Y$ be the row and column variables, respectively. Let

$$
A_{t}=\operatorname{Pr}(X+Y=t, X<Y) \quad \text { and } \quad B_{t}=\operatorname{Pr}(X+Y=t, X>Y) .
$$

for $t=3,4, \ldots, 2 C-1$.

Yamamoto et al. (2013) proposed the SS and CSS models defined by $A_{t}=B_{t}$ and $A_{t}=\Delta B_{t}$, respectively, for $t=3,4, \ldots, 2 C-1$. Note that the parameter $\Delta$ is unspecified. The CSS model with $\Delta=1$ is equivalent to the SS model. Yamamoto et al. (2016) proposed the ESS model defined by $A_{t}=\Delta^{t-2} B_{t}$ for $t=3,4, \ldots, 2 C-1$. The ESS model with $\Delta=1$ is equivalent to the SS model.

To provide a new decomposition theorem for the SS model, we propose the weighted global-sum-symmetry (WGSS) model, defined by

$$
\sum_{t=3}^{2 C-1}(t-2) A_{t}=\sum_{t=3}^{2 C-1}(t-2) B_{t} .
$$


This WGSS model indicates that the weighted sum probability that an observation will fall in one of the cells for which the sum of the row and column levels is $t$ when the row level is less than the column level is equal to the weighted sum probability that an observation will fall in one of the cells for which the sum of the row and column levels is $t$ when the row level is greater than the column level.

It must be noted that the WGSS model is different from the GS model defined by

$$
\sum_{t=3}^{2 C-1} A_{t}=\sum_{t=3}^{2 C-1} B_{t}
$$

This GS model indicates that the sum probability that an observation will fall in one of the cells in the upper right triangle of the table is equal to the sum probability that it will fall in one of the cells in the lower left triangle of the table. The interpretation of the GS model is simpler than that of the WGSS model.

The numbers of degrees of freedom for testing the goodness-of-fit of the SS, CSS, ESS, WGSS, and GS models are $2 C-3,2 C-4,2 C-4,1$, and 1 , respectively. It must be noted that the number of degrees of freedom for the SS model is equal to the sum of those for the ESS and WGSS (or GS) models.

We obtain the following lemma.

Lemma 2.1. The SS model holds if and only if both the ESS and WGSS models hold.

Proof. It is clear that the following necessary condition holds: if the SS model holds, then both the ESS and WGSS models hold. We need to show that the following sufficient condition also holds: if both the ESS and WGSS models hold, then the SS model holds. Since both the ESS and WGSS models hold, the following equality holds:

$$
\sum_{t=3}^{2 C-1}(t-2) \Delta^{t-2} B_{t}=\sum_{t=3}^{2 C-1}(t-2) B_{t} .
$$

Therefore, we obtain $\Delta=1$ (i.e., $A_{t}=B_{t}$ for $t=3,4, \ldots, 2 C-1$ ). The proof is complete. 
Let $f_{i j}$ denote the observed frequency in the $(i, j)$ th cell of the table $(i=1, \ldots, C ; j=1, \ldots, C)$, with a sample size $n\left(=\sum \sum f_{i j}\right)$. We assume multinomial sampling over the cells of the square contingency table. In other words, the observed frequencies $\left\{f_{i j}\right\}$ have a multinomial distribution with parameters that are cell probabilities $\left\{\pi_{i j}\right\}$.

Each model can be tested for goodness-of-fit by, for example, the likelihood ratio chi-squared statistic with the corresponding degrees of freedom. The likelihood ratio chi-squared statistic of the model $\mathrm{M}$ is given by

$$
G^{2}(\mathrm{M})=2 \sum_{i=1}^{C} \sum_{j=1}^{C} f_{i j} \log \left(\frac{f_{i j}}{\hat{e}_{i j}}\right),
$$

where $\hat{e}_{i j}$ is the maximum likelihood estimate (MLE) of the expected frequency $e_{i j}$ under the model M.

Assume that the model $\mathrm{M}_{1}$ holds if and only if both the models $\mathrm{M}_{2}$ and $\mathrm{M}_{3}$ hold and the following asymptotic equivalence holds:

$$
G^{2}\left(\mathrm{M}_{1}\right) \simeq G^{2}\left(\mathrm{M}_{2}\right)+G^{2}\left(\mathrm{M}_{3}\right),
$$

where $G^{2}(\mathrm{M})$ is the likelihood ratio chi-squared statistic of the model M, and the number of degrees of freedom for the model $\mathrm{M}_{1}$ is equal to the sum of those for the models $\mathrm{M}_{2}$ and $\mathrm{M}_{3}$. Darroch and Silvey (1963) mentioned that (i) when equation (1) holds, if both the models $\mathrm{M}_{2}$ and $\mathrm{M}_{3}$ are accepted (at the significance level $\alpha$ ) with high probability, then the model $\mathrm{M}_{1}$ would be accepted. However, (ii) when equation (1) does not hold, it will most likely give rise to an incompatible situation where both the models $\mathrm{M}_{2}$ and $M_{3}$ are accepted with high probability but the $M_{1}$ model is rejected with high probability. This has been exemplified by Darroch and Silvey (1963). Therefore, it is preferable that the decomposition theorem satisfies equation (1).

We obtain the following theorem.

Theorem 2.1. The following asymptotic equivalence holds:

$$
G^{2}(\mathrm{SS}) \simeq G^{2}(\mathrm{ESS})+G^{2}(\mathrm{WGSS}) .
$$

Proof. Let $\boldsymbol{\pi}=\left(\pi_{11}, \pi_{12}, \ldots, \pi_{1 C}, \ldots, \pi_{C 1}, \pi_{C 2}, \ldots, \pi_{C C}\right)^{T}$, and let $\boldsymbol{A}^{T}$ denote the transpose of the matrix (or vector) $\boldsymbol{A}$. Thus, $\boldsymbol{\pi}$ is an $C^{2} \times 1$ vector. Since $\Delta=\pi_{12} / \pi_{21}$ under the ESS model, the ESS model is expressed as

$$
\boldsymbol{h}_{1}(\boldsymbol{\pi})=\mathbf{0}_{2 C-4},
$$


where $\boldsymbol{h}_{1}(\boldsymbol{\pi})=\left(h_{1,4}(\boldsymbol{\pi}), h_{1,5}(\boldsymbol{\pi}), \ldots, h_{1,2 C-1}(\boldsymbol{\pi})\right)^{T}$ with

$$
h_{1, t}(\boldsymbol{\pi})=\left(\pi_{21}\right)^{t-2} A_{t}-\left(\pi_{12}\right)^{t-2} B_{t}=\left(\pi_{21}\right)^{t-2} \sum_{\substack{i<j \\ i+j=t}} \pi_{i j}-\left(\pi_{12}\right)^{t-2} \sum_{\substack{i>j \\ i+j=t}} \sum_{i j},
$$

and $\mathbf{0}_{d}$ is an $d \times 1$ vector, with all components being zero.

The WGSS model is expressed as

$$
\boldsymbol{h}_{2}(\boldsymbol{\pi})=\mathbf{0}_{1},
$$

where

$$
\boldsymbol{h}_{2}(\boldsymbol{\pi})=\sum_{t=3}^{2 C-1}(t-2)\left(A_{t}-B_{t}\right)=\sum_{t=3}^{2 C-1}(t-2)\left[\sum_{\substack{i<j \\ i+j=t}} \pi_{i j}-\sum_{\substack{i>j \\ i+j=t}} \sum_{i j}\right] .
$$

From Lemma 2.1, the SS model is expressed as

$$
\boldsymbol{h}_{3}(\boldsymbol{\pi})=\left(\boldsymbol{h}_{1}(\boldsymbol{\pi})^{T}, \boldsymbol{h}_{2}(\boldsymbol{\pi})^{T}\right)^{T}=\mathbf{0}_{2 C-3} .
$$

Let $\boldsymbol{H}_{s}(\boldsymbol{\pi})(s=1,2,3)$ denote the matrix of partial derivatives of $\boldsymbol{h}_{s}(\boldsymbol{\pi})$ with respect to $\boldsymbol{\pi}$ (i.e., $\boldsymbol{H}_{s}(\boldsymbol{\pi})=\partial \boldsymbol{h}_{s}(\boldsymbol{\pi}) / \partial \boldsymbol{\pi}^{T}$ ). Let $\boldsymbol{\Sigma}(\boldsymbol{\pi})$ be $\operatorname{diag}(\boldsymbol{\pi})-\boldsymbol{\pi} \boldsymbol{\pi}^{T}$, where $\operatorname{diag}(\boldsymbol{\pi})$ is a diagonal matrix, with the $i$ th component of $\boldsymbol{\pi}$ as the $i$ th diagonal element. We denote $\boldsymbol{p}$ as $\boldsymbol{\pi}$, with $\left\{\pi_{i j}\right\}$ replaced by $\left\{p_{i j}\right\}$, where $p_{i j}=f_{i j} / n$. Using the delta method, $\sqrt{n}\left(\boldsymbol{h}_{3}(\boldsymbol{p})-\boldsymbol{h}_{3}(\boldsymbol{\pi})\right)$ has an asymptotically normal distribution, with mean $\mathbf{0}_{C-1}$ and covariance matrix

$$
\boldsymbol{H}_{3}(\boldsymbol{\pi}) \boldsymbol{\Sigma}(\boldsymbol{\pi}) \boldsymbol{H}_{3}(\boldsymbol{\pi})^{T}=\left[\begin{array}{ll}
\boldsymbol{H}_{1}(\boldsymbol{\pi}) \boldsymbol{\Sigma}(\boldsymbol{\pi}) \boldsymbol{H}_{1}(\boldsymbol{\pi})^{T} & \boldsymbol{H}_{1}(\boldsymbol{\pi}) \boldsymbol{\Sigma}(\boldsymbol{\pi}) \boldsymbol{H}_{2}(\boldsymbol{\pi})^{T} \\
\boldsymbol{H}_{2}(\boldsymbol{\pi}) \boldsymbol{\Sigma}(\boldsymbol{\pi}) \boldsymbol{H}_{1}(\boldsymbol{\pi})^{T} & \boldsymbol{H}_{2}(\boldsymbol{\pi}) \boldsymbol{\Sigma}(\boldsymbol{\pi}) \boldsymbol{H}_{2}(\boldsymbol{\pi})^{T}
\end{array}\right] .
$$

All the elements of $\boldsymbol{H}_{1}(\boldsymbol{\pi}) \boldsymbol{\Sigma}(\boldsymbol{\pi}) \boldsymbol{H}_{2}(\boldsymbol{\pi})^{T}$ are equal to 0, under the SS model. This is because we obtain the following equalities:

$$
\frac{\partial h_{1, t}(\boldsymbol{\pi})}{\partial \boldsymbol{\pi}^{T}} \operatorname{diag}(\boldsymbol{\pi}) \frac{\partial \boldsymbol{h}_{2}(\boldsymbol{\pi})}{\partial \boldsymbol{\pi}}=(t-2)\left[\left(\pi_{21}\right)^{t-2} \sum_{\substack{i<j \\ i+j=t}} \sum_{i j}-\left(\pi_{12}\right)^{t-2} \sum_{\substack{i>j \\ i+j=t}} \sum_{i j}\right]
$$

for $t=4, \ldots, 2 C-1$,

$$
\boldsymbol{\pi}^{T} \frac{\partial \boldsymbol{h}_{2}(\boldsymbol{\pi})}{\partial \boldsymbol{\pi}}=\sum_{t=3}^{2 C-1}(t-2)\left[\sum_{\substack{i<j \\ i+j=t}} \pi_{i j}-\sum_{\substack{i>j \\ i+j=t}} \pi_{i j}\right] .
$$


Therefore, we obtain

$$
\begin{aligned}
\boldsymbol{h}_{3}(\boldsymbol{\pi})^{T} & {\left[\boldsymbol{H}_{3}(\boldsymbol{\pi}) \boldsymbol{\Sigma}(\boldsymbol{\pi}) \boldsymbol{H}_{3}(\boldsymbol{\pi})^{T}\right]^{-1} \boldsymbol{h}_{3}(\boldsymbol{\pi}) } \\
= & \boldsymbol{h}_{1}(\boldsymbol{\pi})^{T}\left[\boldsymbol{H}_{1}(\boldsymbol{\pi}) \boldsymbol{\Sigma}(\boldsymbol{\pi}) \boldsymbol{H}_{1}(\boldsymbol{\pi})^{T}\right]^{-1} \boldsymbol{h}_{1}(\boldsymbol{\pi}) \\
& +\boldsymbol{h}_{2}(\boldsymbol{\pi})^{T}\left[\boldsymbol{H}_{2}(\boldsymbol{\pi}) \boldsymbol{\Sigma}(\boldsymbol{\pi}) \boldsymbol{H}_{2}(\boldsymbol{\pi})^{T}\right]^{-1} \boldsymbol{h}_{2}(\boldsymbol{\pi}) .
\end{aligned}
$$

Since the Wald statistic is asymptotically equivalent to the likelihood ratio statistic - see, for example, Rao (1973, Sec. 6e. 3), Darroch and Silvey (1963), and Aitchison (1962) - we obtain Theorem 2.1. The proof is complete.

From Theorem 2.1, we see that while Lemma 2.1 satisfies equation (1), the decomposition theorem of Yamamoto et al. (2016) does not satisfy the equation. Therefore, we believe that Lemma 2.1 is superior to the decomposition theorem of Yamamoto et al. (2016).

\section{Application to data}

First, we consider the dataset in Table 1, taken from Stuart (1953). Table 2 gives the values of $G^{2}$, for each of the SS, ESS, GS, and WGSS models. This table shows that the ESS model fits well, but the other models fit poorly. Table 3 shows MLEs of expected frequencies under the ESS model applied to the data of Table 1. Under the ESS model, the MLE of the parameter $\Delta$ is 0.961 . Therefore, a woman's right eye is estimated to be better than her left eye.

Table 2. Values of the likelihood ratio chi-squared statistic $\left(G^{2}\right)$ for the models applied to the data of Table 1.

\begin{tabular}{ccc}
\hline Models & Degrees of freedom & $G^{2}$ \\
\hline SS & 5 & $15.299^{*}$ \\
ESS & 4 & 5.224 \\
GS & 1 & $11.896^{*}$ \\
WGSS & 1 & $10.065^{*}$ \\
\hline \multicolumn{2}{l}{ The symbol $*$ implies significance at the $5 \%$ level. }
\end{tabular}

We want to identify the cause of the SS model's failure to fit the dataset in Table 1. From Lemma 2.1, we can also infer that the SS model does not hold for the dataset in Table 1 because of the WGSS model rather than the ESS model. In this context, it must be noted that the value of the likelihood ratio chi-squared statistic of the SS model is greater than the sum of those 
Table 3. Maximum likelihood estimates of expected frequencies under the exponential sum-symmetry (ESS) model applied to the data of Table 1.

\begin{tabular}{rrrrrr}
\hline & \multicolumn{5}{c}{ Left eye grades } \\
\cline { 2 - 5 } Right eye grades & $(1)$ & $(2)$ & $(3)$ & $(4)$ & Total \\
\hline$(1)$ & 492 & 179 & 82 & 36 & 789 \\
& $(492)$ & $(188.17)$ & $(76.81)$ & $(38.10)$ & \\
$(2)$ & 205 & 1772 & 362 & 117 & 2456 \\
& $(195.83)$ & $(1772)$ & $(383.14)$ & $(110.91)$ & \\
$(3)$ & 78 & 432 & 1512 & 234 & 2256 \\
& $(83.19)$ & $(411.84)$ & $(1512)$ & $(225.17)$ & \\
$(4)$ & 66 & 124 & 266 & 1520 & 1976 \\
& $(62.92)$ & $(130.09)$ & $(274.83)$ & $(1520)$ & \\
\hline Total & 841 & 2507 & 2222 & 1907 & 7477 \\
\hline
\end{tabular}

of the ESS and GS models, though the value of the likelihood ratio chisquared statistic of the SS model is nearly equal to the sum of those of the ESS and WGSS models.

Next, we consider the artificial dataset in Table 4 . Table 5 gives the values of $G^{2}$ for each of the SS, ESS, GS, and WGSS models.

Table 4. Artificial dataset.

\begin{tabular}{rrrrrr}
\hline & $(1)$ & $(2)$ & $(3)$ & $(4)$ & Total \\
\hline$(1)$ & 100 & 14 & 55 & 85 & 254 \\
$(2)$ & 18 & 100 & 65 & 10 & 193 \\
$(3)$ & 35 & 91 & 100 & 93 & 319 \\
$(4)$ & 57 & 13 & 61 & 100 & 231 \\
\hline Total & 210 & 218 & 281 & 288 & 997 \\
\hline
\end{tabular}

Table 5 shows that the ESS and GS models fit well, but the other models fit poorly. This dataset gives rise to a situation that contradicts the decomposition theorem of Yamamoto et al. (2016). Thus, the SS model does not hold, even though both the ESS and GS models hold. However, this dataset does not give rise to a situation that contradicts Lemma 2.1. From Lemma 2.1, we can infer that the SS model does not hold for the dataset in Table 4 because of the WGSS model rather than the ESS model. 
Table 5. Values of the likelihood ratio chi-squared statistic $\left(G^{2}\right)$, for the models applied to the data of Table 4 .

\begin{tabular}{ccc}
\hline Models & Degrees of freedom & $G^{2}$ \\
\hline SS & 5 & $12.087^{*}$ \\
ESS & 4 & 7.126 \\
GS & 1 & 3.704 \\
WGSS & 1 & $4.969^{*}$ \\
\hline \multicolumn{2}{l}{ The symbol $*$ implies significance at the $5 \%$ level. }
\end{tabular}

\section{Concluding remarks}

This study has established a decomposition theorem in which the SS model holds if and only if both the ESS and WGSS models hold (i.e., Lemma 2.1) and shown that the value of the likelihood ratio chi-squared statistic of the SS model is asymptotically equivalent to the sum of those of the ESS and WGSS models (i.e., Theorem 2.1).

Darroch and Silvey (1963) mentioned that (i) when equation (1) holds, if both the models $\mathrm{M}_{2}$ and $\mathrm{M}_{3}$ are accepted (at the significance level $\alpha$ ) with high probability, then the model $\mathrm{M}_{1}$ would be accepted. However, (ii) when equation (1) does not hold, it will most likely give rise to an incompatible situation where both the models $\mathrm{M}_{2}$ and $\mathrm{M}_{3}$ are accepted with high probability but the $\mathrm{M}_{1}$ model is rejected with high probability. Section 3 exemplifies the aforementioned scenario. Based on the aforementioned finding, we believe that Lemma 2.1 is superior to the decomposition theorem of Yamamoto et al. (2016). On the other hand, the interpretation of the GS model is simpler than that of the WGSS model. In this respect the decomposition theorem of Yamamoto et al. (2016) may be superior to Lemma 2.1.

Yamamoto et al. (2016) obtained the decomposition theorem of the SS model using the extended ESS model they introduced earlier. However, Yamamoto et al. (2016) did not mention whether this decomposition theorem satisfies equation (1). We should verify whether this decomposition theorem satisfies equation (1). If this decomposition theorem does not satisfy equation (1), we must provide a new decomposition theorem that satisfies equation (1). This problem is left for future studies.

\section{Acknowledgements}

The author would like to thank the anonymous reviewers and editors for their comments and suggestions for improving this paper. 


\section{REFERENCES}

Agresti A. (1983a): A simple diagonals-parameter symmetry and quasi-symmetry model. Statistics \& Probability Letters 1: 313-316.

Agresti A. (1983b): Testing marginal homogeneity for ordinal categorical variables. Biometrics 39: 505-510.

Aitchison J. (1962): Large-sample restricted parametric tests. Journal of the Royal Statistical Society: Series B 24: 234-250.

Bhapkar V.P. (1966): A note on the equivalence of two test criteria for hypotheses in categorical data. Journal of the American Statistical Association 61: 228235.

Bhapkar V.P. (1979): On tests of marginal symmetry and quasi-symmetry in two and three-dimensional contingency tables. Biometrics 33: 417-426.

Bishop Y.M., Fienberg S.E., Holland P.W. (2007): Discrete multivariate analysis: theory and practice. Springer Science \& Business Media.

Caussinus H. (1965): Contribution à l'Analyse Statistique des Tableaux de Corrélation. Annales de la Faculté des Sciences de l'Université de Toulouse 29: $77-183$.

Darroch J.N., Silvey S.D. (1963): On testing more than one hypothesis. The Annals of Mathematical Statistics 34: 555-567.

Goodman L.A. (1979): Multiplicative models for square contingency tables with ordered categories. Biometrika 66: 413-418.

McCullagh P. (1978): A class of parametric models for the analysis of square contingency tables with ordered categories. Biometrika 65: 413-418.

Rao C.R. (1973): Linear statistical inference and its applications, 2nd ed. Wiley New York.

Read C.B. (1977): Partitioning chi-square in contingency tables: A teaching approach. Communications in Statistics - Theory and Methods 6: 553-562.

Stuart A. (1953): The estimation and comparison of strengths of association in contingency tables. Biometrika 40: 105-110.

Stuart A. (1955): A test for homogeneity of the marginal distributions in a two-way classification. Biometrika 42: 412-416.

Tomizawa S., Tahata K. (2007): The analysis of symmetry and asymmetry: orthogonality of decomposition of symmetry into quasi-symmetry and marginal symmetry for multi-way tables. Journal de la société française de statistique 148: $3-36$.

Yamamoto K., Tanaka Y., Tomizawa S. (2013): Sum-symmetry model and its orthogonal decomposition for square contingency tables with ordered categories. SUT Journal of Mathematics 49: 121-128.

Yamamoto K., Aizawa M., Tomizawa S. (2016): Decomposition of sum-symmetry model for ordinal square contingency tables. European Journal of Statistics and Probability 4: 12-19. 